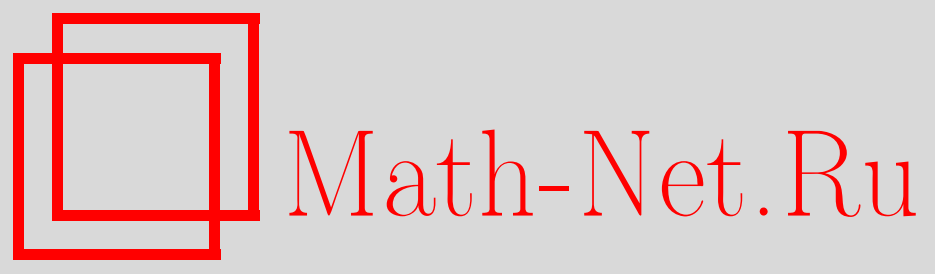

А. М. Зубков, Д. В. Шуваев, Вычисление моментов комбинаторных статистик от перестановочных случайных величин, Дискрет. матем., 2005, том 17, выпуск 2, 3-18

DOI: https://doi.org/10.4213/dm95

Использование Общероссийского математического портала Math-Net.Ru подразумевает, что вы прочитали и согласны с пользовательским соглашением http: //www.mathnet.ru/rus/agreement

Параметры загрузки:

IP : 18.234 .197 .8

26 апреля 2023 г., 16:27:48

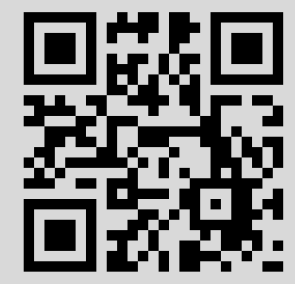


УдК 519.2

\title{
Вычисление моментов комбинаторных статистик от перестановочных случайных величин
}

\author{
() 2005 г. А. М. Зубков, Д. В. Шуваев
}

В настоящей работе рассматриваются статистики, заданные на последовательностях перестановочных случайных величин: число возрастаний, число убываний, число равенств, число двойных возрастаний, двойных убываний, число пиков и число впадин. Получены явные выражения первых моментов таких статистик через простые характеристики совместных распределений.

Работа первого автора выполнена при подлержке грантом НШ-1758.2003.1 программы Президента Российской Федерации поддержки ведущих научных школ и программой РАН «Современные проблемы теоретической математики».

\section{1. Определения и обозначения}

Случайные величины $\xi_{1}, \xi_{2}, \ldots, \xi_{n}$ называются перестановочными, если совместные распределения $\xi_{1}, \xi_{2}, \ldots, \xi_{n}$ и $\xi_{\sigma(1)}, \xi_{\sigma(2)}, \ldots, \xi_{\sigma(n)}$ совпадают при любой перестановке $\sigma(1), \sigma(2), \ldots, \sigma(n)$ чисел $1,2 \ldots, n$.

Примерами распределений наборов $\xi_{1}, \ldots, \xi_{n}$ дискретных перестановочных случайных величин являются равномерное распределение на перестановках $\{1, \ldots, n\}$, равномерное распределение на множествах наборов заданной спецификации и смеси таких распределений. Всюду далее считаем, что $\xi=\left(\xi_{1}, \xi_{2}, \ldots, \xi_{n}\right)-$ последовательность перестановочных случайных величин.

Индикаторную функщию события $A$ будем обозначать $\chi(A): \chi(A)=1$, если $A$ истинно, в противном случае $\chi(A)=0$.

Образуем случайные величины

$$
\begin{aligned}
& R=\sum_{k=1}^{n-1} \chi\left\{\xi_{k}<\xi_{k+1}\right\}, \\
& F=\sum_{k=1}^{n-1} \chi\left\{\xi_{k}>\xi_{k+1}\right\}, \\
& L=\sum_{k=1}^{n-1} \chi\left\{\xi_{k}=\xi_{k+1}\right\},
\end{aligned}
$$


которые равны, соответственно, числу возрастаний, числу убываний и числу равенств в последовательности $\xi_{1}, \ldots, \xi_{n}$. Цель работы - выразить первые моменты распределений $R, L, F$ и некоторых других случайных величин через характеристики распределения случайных величин $\xi_{1}, \xi_{2}, \ldots, \xi_{n}$.

\section{2. Основные результаты}

Распределение трехмерного случайного вектора $(R, F, L)$ сосредоточено на двумерной плоскости, определяемой очевидным равенством

$$
R+F+L=n-1 \text {. }
$$

В теоремах 1-3 приводятся выражения для математического ожидания и компонент ковариационной матрицы вектора $(R, F, L)$. Введем обозначения, которые будут использоваться в работе. Для $n \geqslant 4$ полагаем

$$
\begin{array}{rlrl}
P_{2} & =\mathbf{P}\left\{\xi_{1}=\xi_{2}\right\}, & P_{3} & =\mathbf{P}\left\{\xi_{1}=\xi_{2}=\xi_{3}\right\}, \\
P_{2,2} & =\mathbf{P}\left\{\xi_{1}=\xi_{2}, \xi_{3}=\xi_{4}\right\}, & P_{2}^{*}=\mathbf{P}\left\{\xi_{1}=\xi_{2}<\xi_{3}\right\}-\mathbf{P}\left\{\xi_{1}=\xi_{2}>\xi_{3}\right\} .
\end{array}
$$

Теорема 1. Математическое ожсдание и дисперсия числа возрастаний $R$ равны, соответственно,

$$
\begin{aligned}
& \mathbf{M} R=\frac{(n-1)}{2}\left(1-P_{2}\right), \\
& \mathbf{D} R=\frac{n+1}{12}\left(1-P_{2,2}\right)+\frac{2}{3}(n-2)\left(P_{3}-P_{2,2}\right)+\frac{1}{4}(n-1)^{2}\left(P_{2,2}-P_{2}^{2}\right) .
\end{aligned}
$$

Замечание 1. Если случайные величины $\xi_{1}, \xi_{2}, \ldots, \xi_{n}$ независимы, то

$$
P_{2,2}=P_{2}^{2} \text {, }
$$

и последнее слагаемое в формуле для $\mathrm{D} R$ равно нулю.

Замечание 2. Если $\xi_{1}, \xi_{2}, \ldots, \xi_{n}$ - случайная перестановка, равновероятно выбираемая из множества всех перестановок степени $n$, то

$$
P_{2}=P_{3}=P_{2,2}=0 \text {. }
$$

В этом случае теорема 1 дает формулы

$$
\mathbf{M} R=\frac{n+1}{2}, \quad \mathrm{D} R=\frac{n+1}{12},
$$

согласующиеся с известной предельной теоремой (см. [3], стр. 288).

Теорема 2. Математическое ожидание и дисперсия числа убываний $F$ равны, соответственно,

$$
\mathbf{M} F=\mathbf{M} R, \quad \mathbf{D} F=\mathbf{D} R,
$$

ковариачия $R$ и F равна

$$
\begin{aligned}
\operatorname{cov}(R, F)=-(n-1) & \left(\frac{1-P_{2}}{2}\right)^{2} \\
& +(n-2)\left(\frac{1-P_{2}^{2}}{6}+\frac{P_{2,2}-P_{3}}{3}\right)+(n-2)(n-3)\left(\frac{P_{2,2}-P_{2}^{2}}{4}\right) .
\end{aligned}
$$


Замечание 3. Совпадают не только первые моменты, но и сами распределения числа возрастаний $R$ и числа убываний $F$, поскольку в силу перестановочности случайных величин $\xi_{1}, \xi_{2}, \ldots, \xi_{n}$ отрезки $\left(\xi_{1}, \xi_{2}, \ldots, \xi_{n}\right)$ и $\left(\xi_{n}, \xi_{n-1}, \ldots, \xi_{1}\right)$ распределены одинаково.

Замечание 4. Если случайные величины $\xi_{1}, \xi_{2}, \ldots, \xi_{n}$ независимы, то в силу здмечания 1 последнее слагаемое в правой части выражения для ковариации $R$ и $F$ равно нулю.

Если $\xi_{1}, \xi_{2}, \ldots, \xi_{n}$ реализуют равновероятные перестановки заданной спецификации, то исследование числа убываний $F$ сводится к известной задаче Симона Ньюкомба (см. [2], стр. 254).

Теорема 3. Математическое ожидание и дисперсия числа равенств L равны, соответственно,

$$
\begin{aligned}
\mathbf{M} L & =(n-1) P_{2}, \\
\mathbf{D} L & =-2 \operatorname{cov}(L, R)=-2 \operatorname{cov}(L, F) \\
& =(n-1) P_{2}\left(1-P_{2}\right)+2(n-2)\left(P_{3}-P_{2,2}\right)+(n-2)(n-3)\left(P_{2,2}-P_{2}^{2}\right) .
\end{aligned}
$$

Замечание 5. Утверждения теорем 1, 2 и 3 были опубликованы ранее [1]. Можно также получить утверждения для моментов третьего порядка, одно такое утверждение мы приводим для примера.

При соответствующих предположениях, что $n \geqslant 5,6$, дополнительно полагаем

$$
\begin{aligned}
P_{3,2} & =\mathbf{P}\left\{\xi_{1}=\xi_{2}=\xi_{3}, \xi_{4}=\xi_{5}\right\}, \\
P_{2,2,2} & =\mathbf{P}\left\{\xi_{1}=\xi_{2}, \xi_{3}=\xi_{4}, \xi_{5}=\xi_{6}\right\}
\end{aligned}
$$

Теорема 4. Третий момент числа равенств $L$ равен

$$
\begin{aligned}
& M L^{3}=(n-1) P_{2}+6(n-2) P_{3}+6(n-3) P_{4} \\
&+3(n-2)(n-3) P_{2,2}+6(n-3)(n-4) P_{3,2}+(n-3)(n-4)(n-5) P_{2,2,2} .
\end{aligned}
$$

Введем случайные величины

$$
\begin{aligned}
& \widehat{R R}=\sum_{k=1}^{n-2} \chi\left\{\xi_{k}<\xi_{k+1}<\xi_{k+2}\right\}, \quad \widehat{R L}=\sum_{k=1}^{n-2} \chi\left\{\xi_{k}<\xi_{k+1}=\xi_{k+2}\right\} \\
& \widehat{R F}=\sum_{k=1}^{n-2} \chi\left\{\xi_{k}<\xi_{k+1}>\xi_{k+2}\right\}, \quad \widehat{L R}=\sum_{k=1}^{n-2} \chi\left\{\xi_{k}=\xi_{k+1}<\xi_{k+2}\right\} \\
& \widehat{L L}=\sum_{k=1}^{n-2} \chi\left\{\xi_{k}=\xi_{k+1}=\xi_{k+2}\right\}, \quad \widehat{L F}=\sum_{k=1}^{n-2} \chi\left\{\xi_{k}=\xi_{k+1}>\xi_{k+2}\right\} \\
& \widehat{F R}=\sum_{k=1}^{n-2} \chi\left\{\xi_{k}>\xi_{k+1}<\xi_{k+2}\right\}, \quad \widehat{F L}=\sum_{k=1}^{n-2} \chi\left\{\xi_{k}>\xi_{k+1}=\xi_{k+2}\right\}, \\
& \widehat{F F}=\sum_{k=1}^{n-2} \chi\left\{\xi_{k}>\xi_{k+1}>\xi_{k+2}\right\}
\end{aligned}
$$

Величины $\widehat{R R}, \widehat{R F}, \widehat{F R}, \widehat{F F}$ назовем, соответственно, числом двойных возрастаний, числом пиков, числом впадин, числом двойных убываний в последовательности $\xi_{1}, \xi_{2}, \ldots, \xi_{n}$. 
В [4] для случая, когда $\left(\xi_{1}, \xi_{2}, \ldots, \xi_{n}\right)$ - равновероятная перестановка степени $n$, получены рекурсивные формулы вычисления совместного распределения этих четырех случайных величин.

При соответствующих предположениях, что $n \geqslant 4,5,6$, дополнительно полагаем

$$
\begin{array}{rlrl}
P_{4} & =\mathbf{P}\left\{\xi_{1}=\xi_{2}=\xi_{3}=\xi_{4}\right\}, & P_{3}^{*}=\mathbf{P}\left\{\xi_{1}=\xi_{2}=\xi_{3}<\xi_{4}\right\}-\mathbf{P}\left\{\xi_{1}=\xi_{2}=\xi_{3}>\xi_{4}\right\}, \\
P_{5}=\mathbf{P}\left\{\xi_{1}=\xi_{2}=\xi_{3}=\xi_{4}=\xi_{5}\right\}, & P_{3,3}=\mathbf{P}\left\{\xi_{1}=\xi_{2}=\xi_{3}, \xi_{4}=\xi_{5}=\xi_{6}\right\},
\end{array}
$$

Теорема 5. Математическое ожидание и дисперсия числа двойных возрастаний $\widehat{R R}$ равны, соответственно,

$$
\begin{aligned}
\mathrm{M} \widehat{R R}= & (n-2)\left(\frac{1}{6}-\frac{P_{2}}{2}+\frac{P_{3}}{3}\right), \\
\mathbf{D} \widehat{R R}= & \frac{23 n-37}{180}\left(1-P_{2,2}\right)-\frac{2 n-3}{6} P_{2}\left(1-P_{3}\right)+\frac{7 n-20}{9}\left(P_{3}-P_{2,2}\right) \\
& +\frac{(n-2)^{2}}{4}\left(P_{2,2}-P_{2}^{2}\right)+\frac{7 n-8}{45}\left(P_{2,2}-P_{3}^{2}\right) \\
& +\frac{2 n^{2}-10 n+11}{6}\left(P_{2} P_{3}-P_{3,2}\right)+\frac{7-2 n}{2}\left(P_{4}-P_{3,2}\right) \\
& +\frac{2(n-4)}{5}\left(P_{5}-P_{3,3}\right)+\frac{(n-4)(5 n-7)}{45}\left(P_{3,3}-P_{3}^{2}\right) .
\end{aligned}
$$

Замечание 6. Как и в замечании 3 , легко видеть, что два набора статистик

$$
(\widehat{R R}, \widehat{R L}, \widehat{L F}), \quad(\widehat{F F}, \widehat{L R}, \widehat{F L})
$$

распределены одинаково.

Теорема 6. Математическое ожидание и дисперсия числа двойных равенств $\widehat{L L}$ равны, соответственно,

$$
\begin{aligned}
\mathrm{M} \widehat{L L}= & (n-2) P_{3}, \\
\mathrm{D} \widehat{L L}= & 2(n-4)\left(P_{5}-P_{3,3}\right)+(n-3)(n-4)\left(P_{3,3}-P_{3}^{2}\right) \\
& +3(n-3)\left(P_{4}-P_{3}\right)+(3 n-8) P_{3}\left(1-P_{3}\right) .
\end{aligned}
$$

Замечание 7. Если случайные величины $\xi_{1}, \xi_{2}, \ldots, \xi_{n}$ независимы, то, дополнительно к замечанию 1 ,

$$
P_{2,2,2}=P_{2}^{3}, \quad P_{3,2}=P_{3} P_{2}, \quad P_{3,3}=P_{3}^{2} .
$$

Замечание 8. Если $\xi_{1}, \xi_{2}, \ldots, \xi_{n}-$ случайная перестановка, равновероятно выбираемая из множества всех перестановок, то, дополнительно к замечанию 2,

$$
P_{3}^{*}=P_{4}=P_{5}=P_{3,2}=P_{2,2,2}=P_{3,3}=0 \text {. }
$$

Теорема 7. Математические ожидания числа пиков $\widehat{R F}$ и числа впадин $\widehat{F R}$ равны, соответственно,

$$
\begin{aligned}
& M \widehat{R F}=(n-2)\left(\frac{1}{3}-\frac{P_{2}-P_{2}^{*}}{2}+\frac{P_{3}}{6}\right), \\
& M \widehat{F R}=(n-2)\left(\frac{1}{3}-\frac{P_{2}+P_{2}^{*}}{2}+\frac{P_{3}}{6}\right) .
\end{aligned}
$$


Замечание 9. В силу очевидных равенств

$$
\begin{aligned}
R+F+L & =n-1, \\
\widehat{R R}+\widehat{R F}+\widehat{R L}+\chi\left\{\xi_{n-1}<\xi_{n}\right\} & =\widehat{R R}+\widehat{F R}+\widehat{L R}+\chi\left\{\xi_{1}<\xi_{2}\right\}=R, \\
\widehat{F R}+\widehat{F F}+\widehat{F L}+\chi\left\{\xi_{n-1}>\xi_{n}\right\} & =\widehat{R F}+\widehat{F F}+\widehat{L F}+\chi\left\{\xi_{1}>\xi_{2}\right\}=F
\end{aligned}
$$

статистики $L, \widehat{R L}, \widehat{L R}, \widehat{F L}, \widehat{L F}$ линейно выражаются через $n, R, F, \widehat{R F}, \widehat{F R}, \widehat{R R}, \widehat{F F}$, $\left\{\xi_{1}<\xi_{2}\right\},\left\{\xi_{1}>\xi_{2}\right\},\left\{\xi_{n-1}<\xi_{n}\right\},\left\{\xi_{n-1}>\xi_{n}\right\}$

\section{3. Вычисление вероятностей некоторых событий, связанных с перестановочными случайными величинами}

В настоящем пункте вычисляются вероятности и некоторые вероятностные характеристики, которые будут использованы при доказательстве теорем.

Лемма 1. Если $\xi_{1}, \xi_{2}, \xi_{3}$ - перестановочные случайные величины, то в соответствии с введенными обозначениями

$$
\begin{aligned}
\mathbf{P}\left\{\xi_{1}<\xi_{2}\right\}=\frac{1-P_{2}}{2}, & \mathbf{P}\left\{\xi_{1} \leqslant \xi_{2}\right\}=\frac{1+P_{2}}{2}, \\
\mathbf{P}\left\{\xi_{1}=\xi_{2}<\xi_{3}\right\}+\mathbf{P}\left\{\xi_{1}=\xi_{2}>\xi_{3}\right\} & =P_{2}-P_{3}, \\
\mathbf{P}\left\{\xi_{1}<\xi_{2}<\xi_{3}\right\} & =\frac{1}{6}-\frac{P_{2}}{2}+\frac{P_{3}}{3}, \\
\mathbf{P}\left\{\xi_{1}<\xi_{3}, \xi_{2}<\xi_{3}\right\} & =\frac{1}{3}-\frac{P_{2}-P_{2}^{*}}{2}+\frac{P_{3}}{6}, \\
\mathbf{P}\left\{\xi_{1}<\xi_{2}, \xi_{1}<\xi_{3}\right\} & =\frac{1}{3}-\frac{P_{2}+P_{2}^{*}}{2}+\frac{P_{3}}{6}, \\
\mathbf{P}\left\{\xi_{1} \leqslant \xi_{3}, \xi_{2}<\xi_{3}\right\} & =\frac{1}{3}-\frac{P_{3}}{3}, \\
\mathbf{P}\left\{\xi_{1} \leqslant \xi_{3}, \xi_{2} \leqslant \xi_{3}\right\} & =\frac{1}{3}+\frac{P_{2}-P_{2}^{*}}{2}+\frac{P_{3}}{6} .
\end{aligned}
$$

Замечание 10. Если $\xi_{1}, \xi_{2}, \xi_{3}$ независимы, одинаково распределены, и распределение $\xi_{1}$ симметрично, то есть совпадает с распределением $C-\xi_{1}$ при некоторой постоянной $C$, To

$$
P_{2}^{*}=P_{3}^{*}=0 \text {. }
$$

Замечание 11. Сумма правых частей (4), (6), (7) равна 1; это является следствием перестановочности $\xi_{1}, \xi_{2}, \xi_{3}$ и того, что события $\left\{\xi_{1}<\xi_{3}, \xi_{2}<\xi_{3}\right\},\left\{\xi_{1}<\xi_{2}, \xi_{3} \leqslant \xi_{2}\right\}$, $\left\{\xi_{2} \leqslant \xi_{1}, \xi_{3} \leqslant \xi_{1}\right\}$ попарно не пересекаются, а их объединение дает полное событие, то есть содержит все возможные исходы.

Доказательство леммы 1. Равенства (1) следуют из тождеств

$$
\begin{gathered}
1=\mathbf{P}\left\{\xi_{1}<\xi_{2}\right\}+\mathbf{P}\left\{\xi_{1}=\xi_{2}\right\}+\mathbf{P}\left\{\xi_{1}>\xi_{2}\right\}, \\
\mathbf{P}\left\{\xi_{1}<\xi_{2}\right\}=\mathbf{P}\left\{\xi_{1}>\xi_{2}\right\} .
\end{gathered}
$$


Аналогично выводится (2) из равенства

$$
P_{2}=\mathbf{P}\left\{\xi_{1}=\xi_{2}\right\}=\mathbf{P}\left\{\xi_{1}=\xi_{2}<\xi_{3}\right\}+\mathbf{P}\left\{\xi_{1}=\xi_{2}=\xi_{3}\right\}+\mathbf{P}\left\{\xi_{1}=\xi_{2}>\xi_{3}\right\} .
$$

Равенство (3) следует из (2) и того, что в силу перестановочности случайных величин $\xi_{1}$, $\xi_{2}, \xi_{3}$

$$
\begin{aligned}
1 & =3 ! \mathbf{P}\left\{\xi_{1}<\xi_{2}<\xi_{3}\right\}+3 \mathbf{P}\left\{\xi_{1}=\xi_{2}<\xi_{3}\right\}+3 \mathbf{P}\left\{\xi_{1}=\xi_{2}>\xi_{3}\right\}+\mathbf{P}\left\{\xi_{1}=\xi_{2}=\xi_{3}\right\} \\
& =6 \mathbf{P}\left\{\xi_{1}<\xi_{2}<\xi_{3}\right\}+3 P_{2}-2 P_{3} .
\end{aligned}
$$

Используя (3), (2) и определение $P_{2}^{*}$, получаем (4):

$$
\begin{aligned}
\mathbf{P}\left\{\xi_{1}<\xi_{3}, \xi_{2}<\xi_{3}\right\} & =\mathbf{P}\left\{\xi_{1}<\xi_{2}<\xi_{3}\right\}+\mathbf{P}\left\{\xi_{2}<\xi_{1}<\xi_{3}\right\}+\mathbf{P}\left\{\xi_{1}=\xi_{2}<\xi_{3}\right\} \\
& =2 \mathbf{P}\left\{\xi_{1}<\xi_{2}<\xi_{3}\right\}+\frac{P_{2}-P_{3}+P_{2}^{*}}{2} \\
& =\frac{1}{3}-\frac{P_{2}}{2}+\frac{P_{3}}{6}+\frac{P_{2}^{*}}{2}
\end{aligned}
$$

Аналогично получаем равенство (5):

$$
\begin{aligned}
\mathbf{P}\left\{\xi_{1}<\xi_{2}, \xi_{1}<\xi_{3}\right\} & =2 \mathbf{P}\left\{\xi_{1}<\xi_{2}<\xi_{3}\right\}+\frac{P_{2}-P_{3}-P_{2}^{*}}{2} \\
& =\frac{1}{3}-\frac{P_{2}}{2}+\frac{P_{3}}{6}-\frac{P_{2}^{*}}{2} .
\end{aligned}
$$

Равенство (6) следует из (4), (2) и определения $P_{2}^{*}$ :

$$
\begin{aligned}
\mathbf{P}\left\{\xi_{1} \leqslant \xi_{3}, \xi_{2}<\xi_{3}\right\} & =\mathbf{P}\left\{\xi_{1}<\xi_{3}, \xi_{2}<\xi_{3}\right\}+\mathbf{P}\left\{\xi_{1}=\xi_{3}>\xi_{2}\right\} \\
& =\frac{1}{3}-\frac{P_{2}-P_{2}^{*}}{2}+\frac{P_{3}}{6}+\frac{P_{2}-P_{3}-P_{2}^{*}}{2} \\
& =\frac{1}{3}-\frac{P_{3}}{3} .
\end{aligned}
$$

Наконец, (7) выводится из (4) и (2):

$$
\begin{aligned}
\mathbf{P}\left\{\xi_{1} \leqslant \xi_{3}, \xi_{2} \leqslant \xi_{3}\right\}= & \mathbf{P}\left\{\xi_{1}<\xi_{3}, \xi_{2}<\xi_{3}\right\}+\mathbf{P}\left\{\xi_{1}<\xi_{3}=\xi_{2}\right\} \\
& +\mathbf{P}\left\{\xi_{1}=\xi_{3}>\xi_{2}\right\}+\mathbf{P}\left\{\xi_{1}=\xi_{2}=\xi_{3}\right\} \\
= & \frac{1}{3}-\frac{P_{2}-P_{2}^{*}}{2}+\frac{P_{3}}{6}+\left(P_{2}-P_{3}-P_{2}^{*}\right)+P_{3} \\
= & \frac{1}{3}+\frac{P_{2}-P_{2}^{*}}{2}+\frac{P_{3}}{3} .
\end{aligned}
$$

Следствие 1. Для перестановочных случайных величин $\xi_{1}, \xi_{2}, \xi_{3}$ справедливы равенства

$$
\begin{aligned}
D & =\mathbf{D} \chi\left\{\xi_{1} \leqslant \xi_{2}\right\}=\mathbf{D} \chi\left\{\xi_{1}<\xi_{2}\right\}=\frac{1-P_{2}^{2}}{4}, \\
c_{\leqslant \leqslant} & =\operatorname{cov}\left(\chi\left\{\xi_{1} \leqslant \xi_{3}\right\}, \chi\left\{\xi_{2} \leqslant \xi_{3}\right\}\right)=\frac{1-P_{2}^{2}}{12}-\frac{P_{2}^{2}-P_{3}}{6}-\frac{P_{2}^{*}}{2}, \\
c_{\leqslant<} & =\operatorname{cov}\left(\chi\left\{\xi_{1} \leqslant \xi_{3}\right\}, \chi\left\{\xi_{2}<\xi_{3}\right\}\right)=\frac{1-P_{2}^{2}}{12}+\frac{P_{2}^{2}-P_{3}}{3}, \\
c_{<<} & =\operatorname{cov}\left(\chi\left\{\xi_{1}<\xi_{3}\right\}, \chi\left\{\xi_{2}<\xi_{3}\right\}\right)=\frac{1-P_{2}^{2}}{12}-\frac{P_{2}^{2}-P_{3}}{6}+\frac{P_{2}^{*}}{2} .
\end{aligned}
$$


Доказательство. Равенства (8) непосредственно следуют из (1). Далее, из (7), (1) и (4) выводим (9):

$$
\begin{aligned}
\operatorname{cov}\left(\chi\left\{\xi_{1} \leqslant \xi_{3}\right\}, \chi\left\{\xi_{2} \leqslant \xi_{3}\right\}\right) & =\mathbf{P}\left\{\xi_{1} \leqslant \xi_{3}, \xi_{2} \leqslant \xi_{3}\right\}-\mathbf{P}\left\{\xi_{1} \leqslant \xi_{3}\right\} \mathbf{P}\left\{\xi_{2} \leqslant \xi_{3}\right\} \\
& =\frac{1}{3}+\frac{P_{2}-P_{2}^{*}}{2}+\frac{P_{3}}{6}-\frac{\left(1+P_{2}\right)^{2}}{4} \\
& =\frac{1}{12}-\frac{P_{2}^{2}}{4}+\frac{P_{3}}{6}-\frac{P_{2}^{*}}{2} .
\end{aligned}
$$

Аналогично доказываются формулы (10) и (11):

$$
\begin{aligned}
\operatorname{cov}\left(\chi\left\{\xi_{1} \leqslant \xi_{3}\right\}, \chi\left\{\xi_{2}<\xi_{3}\right\}\right) & =\frac{1}{3}-\frac{P_{3}}{3}-\frac{1-P_{2}^{2}}{4}=\frac{1}{12}+\frac{P_{2}^{2}}{4}-\frac{P_{3}}{3} \\
\operatorname{cov}\left(\chi\left\{\xi_{1}<\xi_{3}\right\}, \chi\left\{\xi_{2}<\xi_{3}\right\}\right) & =\frac{1}{3}-\frac{P_{2}-P_{2}^{*}}{2}+\frac{P_{3}}{6}-\frac{\left(1-P_{2}\right)^{2}}{4} \\
& =\frac{1}{12}-\frac{P_{2}^{2}}{4}+\frac{P_{3}}{6}+\frac{P_{2}^{*}}{2}
\end{aligned}
$$

Замечание 12. Если случайные величины $\xi_{1}, \xi_{2}, \xi_{3}$ независимы и имеют равномерное распределение на $\{1, \ldots, m\}$, то

$$
P_{2}=1 / m, \quad P_{3}=1 / m^{2}, \quad P_{2}^{*}=0,
$$

и поэтому

$$
\begin{aligned}
\mathbf{D} \chi\left\{\xi_{1} \leqslant \xi_{2}\right\} & =\mathbf{D} \chi\left\{\xi_{1}<\xi_{2}\right\}=\frac{m^{2}-1}{4 m^{2}} \\
\operatorname{cov}\left(\chi\left\{\xi_{1} \leqslant \xi_{3}\right\}, \chi\left\{\xi_{2} \leqslant \xi_{3}\right\}\right) & =\operatorname{cov}\left(\chi\left\{\xi_{1} \leqslant \xi_{3}\right\}, \chi\left\{\xi_{2}<\xi_{3}\right\}\right) \\
& =\operatorname{cov}\left(\chi\left\{\xi_{1}<\xi_{3}\right\}, \chi\left\{\xi_{2}<\xi_{3}\right\}\right)=\frac{m^{2}-1}{12 m^{2}}
\end{aligned}
$$

Лемма 2. Если $\xi_{1}, \xi_{2}, \xi_{3}, \xi_{4}$ - перестановочные случайные величины, то

$$
\begin{aligned}
& \mathbf{P}\left\{\xi_{1}<\xi_{2}, \xi_{3}<\xi_{4}\right\}=\frac{1}{4}-\frac{P_{2}}{2}+\frac{P_{2,2}}{4} \\
& \mathbf{P}\left\{\xi_{1} \leqslant \xi_{2}, \xi_{3}<\xi_{4}\right\}=\frac{1}{4}-\frac{P_{2,2}}{4} \\
& \mathbf{P}\left\{\xi_{1} \leqslant \xi_{2}, \xi_{3} \leqslant \xi_{4}\right\}=\frac{1}{4}+\frac{P_{2}}{2}+\frac{P_{2,2}}{4} .
\end{aligned}
$$

Доказательство. Из тождества

$$
\begin{aligned}
\mathbf{P}\left\{\xi_{1}=\xi_{2}\right\} & =\mathbf{P}\left\{\xi_{1}=\xi_{2}, \xi_{3}<\xi_{4}\right\}+\mathbf{P}\left\{\xi_{1}=\xi_{2}, \xi_{3}=\xi_{4}\right\}+\mathbf{P}\left\{\xi_{1}=\xi_{2}, \xi_{3}>\xi_{4}\right\} \\
& =2 \mathbf{P}\left\{\xi_{1}=\xi_{2}, \xi_{3}<\xi_{4}\right\}+\mathbf{P}\left\{\xi_{1}=\xi_{2}, \xi_{3}=\xi_{4}\right\}
\end{aligned}
$$

следует равенство

$$
\mathbf{P}\left\{\xi_{1}=\xi_{2}, \xi_{3}<\xi_{4}\right\}=\frac{P_{2}-P_{2,2}}{2}
$$


Из аналогичного тождества

$$
\mathbf{P}\left\{\xi_{1}<\xi_{2}\right\}=2 \mathbf{P}\left\{\xi_{1}<\xi_{2}, \xi_{3}<\xi_{4}\right\}+\mathbf{P}\left\{\xi_{1}<\xi_{2}, \xi_{3}=\xi_{4}\right\}
$$

и из (17) получаем, что

$$
\begin{aligned}
\mathbf{P}\left\{\xi_{1}<\xi_{2}, \xi_{3}<\xi_{4}\right\} & =\frac{1}{2}\left(\frac{1-P_{2}}{2}-\frac{P_{2}-P_{2,2}}{2}\right) \\
& =\frac{1}{4}-\frac{P_{2}}{2}+\frac{P_{2,2}}{4}
\end{aligned}
$$

Равенство (15) получается сложением правых частей (14) и (17).

Докажем (16):

$$
\begin{aligned}
\mathbf{P}\left\{\xi_{1} \leqslant \xi_{2}, \xi_{3} \leqslant \xi_{4}\right\} & =\mathbf{P}\left\{\xi_{1} \leqslant \xi_{2}, \xi_{3}<\xi_{4}\right\}+\mathbf{P}\left\{\xi_{1} \leqslant \xi_{2}, \xi_{3}=\xi_{4}\right\} \\
& =\frac{1-P_{2,2}}{4}+\frac{P_{2}-P_{2,2}}{2}+P_{2,2} \\
& =\frac{1+2 P_{2}+P_{2,2}}{4}
\end{aligned}
$$

Из леммы 2 получаем следующее утверждение.

Следствие 2. Для перестановочных случайных величин $\xi_{1}, \xi_{2}, \xi_{3}, \xi_{4}$ справедливы равенствa

$$
\begin{aligned}
c_{4} & =\operatorname{cov}\left(\chi\left\{\xi_{1} \leqslant \xi_{2}\right\}, \chi\left\{\xi_{3} \leqslant \xi_{4}\right\}\right) \\
& =-\operatorname{cov}\left(\chi\left\{\xi_{1} \leqslant \xi_{2}\right\}, \chi\left\{\xi_{3}<\xi_{4}\right\}\right) \\
& =\operatorname{cov}\left(\chi\left\{\xi_{1}<\xi_{2}\right\}, \chi\left\{\xi_{3}<\xi_{4}\right\}\right) \\
& =\frac{P_{2,2}-P_{2}^{2}}{4} .
\end{aligned}
$$

Доказательство. Докажем последнее из трех равенств:

$$
\begin{aligned}
\operatorname{cov}\left(\chi\left\{\xi_{1}<\xi_{2}\right\}, \chi\left\{\xi_{3}<\xi_{4}\right\}\right) & =\mathbf{P}\left\{\xi_{1}<\xi_{2}, \xi_{3}<\xi_{4}\right\}-\mathbf{P}\left\{\xi_{1}<\xi_{2}\right\} \mathbf{P}\left\{\xi_{3}<\xi_{4}\right\} \\
& =\frac{1-2 P_{2}+P_{2,2}}{4}-\left(\frac{1-P_{2}}{2}\right)^{2} \\
& =\frac{P_{2,2}-P_{2}^{2}}{4} .
\end{aligned}
$$

Два других равенства следуют из очевидного тождества

$$
\chi\left\{\xi_{1} \leqslant \xi_{2}\right\}=1-\chi\left\{\xi_{2}<\xi_{1}\right\}
$$

и некоррелированности произвольных случайных величин с константами.

Лемма 3. Если $\xi_{1}, \xi_{2}, \xi_{3}, \xi_{4}$ - перестановочные случайные величины, то в соответствии 
с введенными обозначениями

$$
\begin{aligned}
\mathbf{P}\left\{\xi_{1}=\xi_{2}<\xi_{3}=\xi_{4}\right\} & =\frac{P_{2,2}-P_{4}}{2} \\
\mathbf{P}\left\{\xi_{1}=\xi_{2}=\xi_{3}>\xi_{4}\right\} & =\frac{P_{3}-P_{4}-P_{3}^{*}}{2} \\
\mathbf{P}\left\{\xi_{1}=\xi_{2}=\xi_{3}<\xi_{4}\right\} & =\frac{P_{3}-P_{4}+P_{3}^{*}}{2} \\
2 \mathbf{P}\left\{\xi_{1}=\xi_{2}<\xi_{3}<\xi_{4}\right\}+\mathbf{P}\left\{\xi_{1}<\xi_{2}=\xi_{3}<\xi_{4}\right\} & =\frac{\left(P_{2}-P_{2,2}\right)+\left(P_{2}^{*}-P_{3}^{*}\right)}{2}+P_{4}-P_{3} \\
2 \mathbf{P}\left\{\xi_{1}<\xi_{2}<\xi_{3}=\xi_{4}\right\}+\mathbf{P}\left\{\xi_{1}<\xi_{2}=\xi_{3}<\xi_{4}\right\} & =\frac{\left(P_{2}-P_{2,2}\right)-\left(P_{2}^{*}-P_{3}^{*}\right)}{2}+P_{4}-P_{3} \\
\mathbf{P}\left\{\xi_{1}<\xi_{2}<\xi_{3}<\xi_{4}\right\} & =\frac{1}{24}-\frac{P_{2}}{4}+\frac{P_{3}}{3}+\frac{P_{2,2}}{8}-\frac{P_{4}}{4}
\end{aligned}
$$

Доказательство. Равенство (19) следует из перестановочности $\xi_{1}, \xi_{2}, \xi_{3}, \xi_{4}$ и очевидного тождества

$$
\begin{aligned}
P_{2,2} & =\mathbf{P}\left\{\xi_{1}=\xi_{2}, \xi_{3}=\xi_{4}\right\} \\
& =P_{4}+\mathbf{P}\left\{\xi_{1}=\xi_{2}<\xi_{3}=\xi_{4}\right\}+\mathbf{P}\left\{\xi_{1}=\xi_{2}>\xi_{3}=\xi_{4}\right\}
\end{aligned}
$$

Равенства (20) и (21) следуют из определения $P_{3}^{*}$ и тождества

$$
\begin{aligned}
P_{3} & =\mathbf{P}\left\{\xi_{1}=\xi_{2}=\xi_{3}\right\} \\
& =P_{4}+\mathbf{P}\left\{\xi_{1}=\xi_{2}=\xi_{3}<\xi_{4}\right\}+\mathbf{P}\left\{\xi_{1}=\xi_{2}=\xi_{3}>\xi_{4}\right\}
\end{aligned}
$$

Выполняется цепочка равенств

$$
\begin{aligned}
\mathbf{P}\left\{\xi_{1}=\xi_{2}<\xi_{3}\right\}= & \mathbf{P}\left\{\xi_{1}=\xi_{2}<\xi_{3}<\xi_{4}\right\}+\mathbf{P}\left\{\xi_{1}=\xi_{2}<\xi_{3}=\xi_{4}\right\} \\
& +\mathbf{P}\left\{\xi_{1}=\xi_{2}<\xi_{3}>\xi_{4}\right\} \\
= & \mathbf{P}\left\{\xi_{1}=\xi_{2}<\xi_{3}<\xi_{4}\right\}+\mathbf{P}\left\{\xi_{1}=\xi_{2}<\xi_{3}=\xi_{4}\right\} \\
& +\mathbf{P}\left\{\xi_{1}=\xi_{2}<\xi_{4}<\xi_{3}\right\}+\mathbf{P}\left\{\xi_{1}=\xi_{2}=\xi_{4}<\xi_{3}\right\} \\
& +\mathbf{P}\left\{\xi_{4}<\xi_{1}=\xi_{2}<\xi_{3}\right\} .
\end{aligned}
$$

С учетом (19) и (21) выражение в правой части цепочки преобразуется к виду

$$
2 \mathbf{P}\left\{\xi_{1}=\xi_{2}<\xi_{3}<\xi_{4}\right\}+\frac{P_{2,2}-P_{4}}{2}+\frac{P_{3}-P_{4}+P_{3}^{*}}{2}+\mathbf{P}\left\{\xi_{1}<\xi_{2}=\xi_{3}<\xi_{4}\right\},
$$

но согласно определению $P_{2}^{*}$ левая часть цепочки есть $\left(P_{2}+P_{2}^{*}-P_{3}\right) / 2$. Равенство левой и правой частей цепочки после приведения подобных членов доказывает формулу (22). Формула (23) выводится аналогичным образом из равенства

$$
\begin{aligned}
\mathbf{P}\left\{\xi_{2}<\xi_{3}=\xi_{4}\right\}= & \mathbf{P}\left\{\xi_{1}<\xi_{2}<\xi_{3}=\xi_{4}\right\}+\mathbf{P}\left\{\xi_{1}=\xi_{2}<\xi_{3}=\xi_{4}\right\} \\
& +\mathbf{P}\left\{\xi_{2}<\xi_{1}<\xi_{3}=\xi_{4}\right\}+\mathbf{P}\left\{\xi_{2}<\xi_{1}=\xi_{3}=\xi_{4}\right\} \\
& +\mathbf{P}\left\{\xi_{2}<\xi_{3}=\xi_{4}<\xi_{1}\right\}
\end{aligned}
$$


с учетом формулы

$$
\mathbf{P}\left\{\xi_{2}<\xi_{3}=\xi_{4}\right\}=\frac{P_{2}-P_{2}^{*}-P_{3}}{2}
$$

и формул (19), (20). Докажем (24). В силу перестановочности $\xi_{1}, \xi_{2}, \xi_{3}, \xi_{4}$ выполняется тождество

$$
\begin{aligned}
1=4 & ! \mathbf{P}\left\{\xi_{1}<\xi_{2}<\xi_{3}<\xi_{4}\right\} \\
& +12\left(\mathbf{P}\left\{\xi_{1}=\xi_{2}<\xi_{3}<\xi_{4}\right\}+\mathbf{P}\left\{\xi_{1}<\xi_{2}=\xi_{3}<\xi_{4}\right\}+\mathbf{P}\left\{\xi_{1}<\xi_{2}<\xi_{3}=\xi_{4}\right\}\right) \\
& +4\left(\mathbf{P}\left\{\xi_{1}=\xi_{2}=\xi_{3}<\xi_{4}\right\}+\mathbf{P}\left\{\xi_{1}<\xi_{2}=\xi_{3}=\xi_{4}\right\}\right) \\
& +6 \mathbf{P}\left\{\xi_{1}=\xi_{2}<\xi_{3}=\xi_{4}\right\}+\mathbf{P}\left\{\xi_{1}=\xi_{2}=\xi_{3}=\xi_{4}\right\} .
\end{aligned}
$$

Рассмотрим сумму в правой части равенства. Группа слагаемых, перед которыми стоит коэффициент 12 , равна, очевидно, полусумме

$$
2 \mathbf{P}\left\{\xi_{1}=\xi_{2}<\xi_{3}<\xi_{4}\right\}+\mathbf{P}\left\{\xi_{1}<\xi_{2}=\xi_{3}<\xi_{4}\right\}
$$

и

$$
2 \mathbf{P}\left\{\xi_{1}<\xi_{2}<\xi_{3}=\xi_{4}\right\}+\mathbf{P}\left\{\xi_{1}<\xi_{2}=\xi_{3}<\xi_{4}\right\}
$$

и выражается с помощью (22) и (23). Группа слагаемых перед которыми стоит коэффициент 4, выражается через (20) и (21), слагаемое с коэффициентом 6 выражается через (19), последнее слагаемое есть $P_{4}$. В результате указанных преобразований и приведения подобных членов получаем (24).

Лемма доказана.

Лемма 4. Если $\xi_{1}, \xi_{2}, \xi_{3}, \xi_{4}, \xi_{5}$ - перестановочные случайные величины, то в соответствии с введенными обозначениями

$$
\begin{gathered}
\mathbf{P}\left\{\xi_{1}=\xi_{2}=\xi_{3}, \xi_{4}<\xi_{5}\right\}=\frac{1}{2}\left(P_{3}-P_{3,2}\right), \\
\mathbf{P}\left\{\xi_{1}=\xi_{2}=\xi_{3}=\xi_{4}<\xi_{5}\right\}+\mathbf{P}\left\{\xi_{1}=\xi_{2}=\xi_{3}=\xi_{4}>\xi_{5}\right\}=P_{4}-P_{5}, \\
\mathbf{P}\left\{\xi_{1}=\xi_{2}=\xi_{3}<\xi_{4}=\xi_{5}\right\}+\mathbf{P}\left\{\xi_{1}=\xi_{2}=\xi_{3}>\xi_{4}=\xi_{5}\right\}=P_{3,2}-P_{5}, \\
\mathbf{P}\left\{\xi_{1}<\xi_{2}<\xi_{3}<\xi_{4}<\xi_{5}\right\}=\frac{1}{120}-\frac{P_{2}}{12}+\frac{P_{3}}{6}-\frac{P_{4}}{4}+\frac{P_{5}}{5}+\frac{P_{2,2}}{8}-\frac{P_{3,2}}{6}
\end{gathered}
$$

Доказательство. В силу перестановочности

$$
\mathbf{P}\left\{\xi_{1}=\xi_{2}=\xi_{3}, \xi_{4}<\xi_{5}\right\}=\mathbf{P}\left\{\xi_{1}=\xi_{2}=\xi_{3}, \xi_{4}>\xi_{5}\right\}
$$

поэтому

$$
2 \mathbf{P}\left\{\xi_{1}=\xi_{2}=\xi_{3}, \xi_{4}<\xi_{5}\right\}+\mathbf{P}\left\{\xi_{1}=\xi_{2}=\xi_{3}, \xi_{4}=\xi_{5}\right\}=\mathbf{P}\left\{\xi_{1}=\xi_{2}=\xi_{3}\right\}
$$

Из последнего равенства следует (25). Равенства (26) и (27) следуют непосредственно из определения величин $P_{3,2}, P_{4}, P_{5}$. Равенство (28) докажем, исходя из следующего 
тождества:

$$
\begin{array}{rl}
1=5 & \mathbf{P}\left\{\xi_{1}<\xi_{2}<\xi_{3}<\xi_{4}<\xi_{5}\right\}+\frac{5 !}{2} \mathbf{P}\left\{\xi_{1}=\xi_{2}<\xi_{3}<\xi_{4}<\xi_{5}\right\} \\
& +\frac{5 !}{2} \mathbf{P}\left\{\xi_{1}<\xi_{2}=\xi_{3}<\xi_{4}<\xi_{5}\right\}+\frac{5 !}{2} \mathbf{P}\left\{\xi_{1}<\xi_{2}<\xi_{3}=\xi_{4}<\xi_{5}\right\} \\
& +\frac{5 !}{2} \mathbf{P}\left\{\xi_{1}<\xi_{2}<\xi_{3}<\xi_{4}=\xi_{5}\right\}+\frac{5 !}{3 !} \mathbf{P}\left\{\xi_{1}=\xi_{2}=\xi_{3}<\xi_{4}<\xi_{5}\right\} \\
& +\frac{5 !}{2^{2}} \mathbf{P}\left\{\xi_{1}=\xi_{2}<\xi_{3}=\xi_{4}<\xi_{5}\right\}+\frac{5 !}{2^{2}} \mathbf{P}\left\{\xi_{1}=\xi_{2}<\xi_{3}<\xi_{4}=\xi_{5}\right\} \\
& +\frac{5 !}{3 !} \mathbf{P}\left\{\xi_{1}<\xi_{2}=\xi_{3}=\xi_{4}<\xi_{5}\right\}+\frac{5 !}{2^{2}} \mathbf{P}\left\{\xi_{1}<\xi_{2}=\xi_{3}<\xi_{4}=\xi_{5}\right\} \\
& +\frac{5 !}{3 !} \mathbf{P}\left\{\xi_{1}<\xi_{2}<\xi_{3}=\xi_{4}=\xi_{5}\right\}+\frac{5 !}{4 !} \mathbf{P}\left\{\xi_{1}=\xi_{2}=\xi_{3}=\xi_{4}<\xi_{5}\right\} \\
& +\frac{5 !}{2 ! 3 !} \mathbf{P}\left\{\xi_{1}=\xi_{2}=\xi_{3}<\xi_{4}=\xi_{5}\right\}+\frac{5 !}{2 ! 3 !} \mathbf{P}\left\{\xi_{1}=\xi_{2}<\xi_{3}=\xi_{4}=\xi_{5}\right\} \\
& +\frac{5 !}{4 !} \mathbf{P}\left\{\xi_{1}<\xi_{2}=\xi_{3}=\xi_{4}=\xi_{5}\right\}+\mathbf{P}\left\{\xi_{1}=\xi_{2}=\xi_{3}=\xi_{4}=\xi_{5}\right\} .
\end{array}
$$

Преобразуем правую часть тождества, разбив для удобства всю сумму на отдельные группы слагаемых с одинаковыми коэффициентами (точнее, с коэффициентами, записанными одинаковым образом). Слагаемые первой группы определяются искомой величиной. Слагаемые второй группы (с коэффициентами 5!/2) могут быть описаны следующим образом: среди $\xi_{1}, \xi_{2}, \xi_{3}, \xi_{4}, \xi_{5}$ найдется ровно четыре различных значения. Заметим, что если $\xi_{1}<\xi_{2}<\xi_{3}<\xi_{4}$, то значение $\xi_{5}$ либо совпадает с одним из $\xi_{1}, \xi_{2}, \xi_{3}, \xi_{4}$, либо может быть вставлено в строго возрастающую цепочку $\xi_{1}<\xi_{2}<\xi_{3}<\xi_{4}$ одним из пяти способов. Из этого следует равенство

$$
\begin{aligned}
& \mathbf{P}\left\{\xi_{1}=\xi_{2}<\xi_{3}<\xi_{4}<\xi_{5}\right\}+\mathbf{P}\left\{\xi_{1}<\xi_{2}=\xi_{3}<\xi_{4}<\xi_{5}\right\}+\mathbf{P}\left\{\xi_{1}<\xi_{2}<\xi_{3}=\xi_{4}<\xi_{5}\right\} \\
& \quad+\mathbf{P}\left\{\xi_{1}<\xi_{2}<\xi_{3}<\xi_{4}=\xi_{5}\right\}=\mathbf{P}\left\{\xi_{1}<\xi_{2}<\xi_{3}<\xi_{4}\right\}-5 \mathbf{P}\left\{\xi_{1}<\xi_{2}<\xi_{3}<\xi_{4}<\xi_{5}\right\}
\end{aligned}
$$

в котором с учетом свойства перестановочности мы пренебрегли различными перестановками индексов. Рассмотрим третью группу слагаемых (с коэффициентами 5!/3!), их можно описать следующим образом: среди $\xi_{1}, \xi_{2}, \xi_{3}, \xi_{4}, \xi_{5}$ найдется три равных и найдется три попарно различных значения. Из равенств (25), (26) и очевидного тождества

$$
\begin{aligned}
\mathbf{P}\left\{\xi_{1}=\xi_{2}=\xi_{3}, \xi_{4}<\xi_{5}\right\}= & \mathbf{P}\left\{\xi_{1}=\xi_{2}=\xi_{3}<\xi_{4}<\xi_{5}\right\} \\
& +\mathbf{P}\left\{\xi_{1}=\xi_{2}=\xi_{3}=\xi_{4}<\xi_{5}\right\} \\
& +\mathbf{P}\left\{\xi_{4}<\xi_{1}=\xi_{2}=\xi_{3}<\xi_{5}\right\} \\
& +\mathbf{P}\left\{\xi_{4}<\xi_{5}=\xi_{1}=\xi_{2}<\xi_{3}\right\} \\
& +\mathbf{P}\left\{\xi_{4}<\xi_{5}<\xi_{1}=\xi_{2}<\xi_{3}\right\}
\end{aligned}
$$

получим формулу для преобразования третьей группы слагаемых:

$$
\begin{aligned}
\mathbf{P}\left\{\xi_{1}=\xi_{2}=\xi_{3}<\xi_{4}<\xi_{5}\right\} & +\mathbf{P}\left\{\xi_{1}<\xi_{2}=\xi_{3}=\xi_{4}<\xi_{5}\right\} \\
& +\mathbf{P}\left\{\xi_{1}<\xi_{2}<\xi_{3}=\xi_{4}=\xi_{5}\right\}=\left(P_{3}-P_{3,2}\right) / 2-P_{4}+P_{5} .
\end{aligned}
$$

Слагаемые четвертой группы описываются следующим образом: среди $\xi_{1}, \xi_{2}, \xi_{3}, \xi_{4}, \xi_{5}$ найдется две пары равных значений и найдется три попарно различных значения. Найдем 
выражение для преобразования этой группы. Последовательно получаем:

$$
\begin{aligned}
P_{2,2}= & \mathbf{P}\left\{\xi_{1}=\xi_{2}, \xi_{3}=\xi_{4}\right\} \\
= & \mathbf{P}\left\{\xi_{1}=\xi_{2}<\xi_{5}, \xi_{3}=\xi_{4}\right\}+P_{3,2}+\mathbf{P}\left\{\xi_{1}=\xi_{2}>\xi_{5}, \xi_{3}=\xi_{4}\right\} \\
= & \mathbf{P}\left\{\xi_{1}=\xi_{2}<\xi_{5}<\xi_{3}=\xi_{4}\right\}+\mathbf{P}\left\{\xi_{1}=\xi_{2}<\xi_{3}=\xi_{4}=\xi_{5}\right\} \\
& +\mathbf{P}\left\{\xi_{1}=\xi_{2}<\xi_{3}=\xi_{4}<\xi_{5}\right\}+\mathbf{P}\left\{\xi_{1}=\xi_{2}=\xi_{3}=\xi_{4}<\xi_{5}\right\} \\
& +\mathbf{P}\left\{\xi_{3}=\xi_{4}<\xi_{1}=\xi_{2}<\xi_{5}\right\}+P P_{3,2}+\mathbf{P}\left\{\xi_{1}=\xi_{2}>\xi_{5}>\xi_{3}=\xi_{4}\right\} \\
& +\mathbf{P}\left\{\xi_{1}=\xi_{2}>\xi_{3}=\xi_{4}=\xi_{5}\right\}+\mathbf{P}\left\{\xi_{1}=\xi_{2}>\xi_{3}=\xi_{4}>\xi_{5}\right\} \\
& +\mathbf{P}\left\{\xi_{1}=\xi_{2}=\xi_{3}=\xi_{4}>\xi_{5}\right\}+\mathbf{P}\left\{\xi_{3}=\xi_{4}>\xi_{1}=\xi_{2}>\xi_{5}\right\} .
\end{aligned}
$$

Используя перестановочность, переставим индексы в единообразном порядке и продолжим преобразования с учетом формул (27), (26):

$$
\begin{aligned}
& P_{2,2}=2( \mathbf{P}\left\{\xi_{1}=\xi_{2}<\xi_{3}<\xi_{4}=\xi_{5}\right\}+\mathbf{P}\left\{\xi_{1}=\xi_{2}<\xi_{3}=\xi_{4}<\xi_{5}\right\} \\
&\left.+\mathbf{P}\left\{\xi_{1}=\xi_{2}>\xi_{3}=\xi_{4}>\xi_{5}\right\}\right)+\mathbf{P}\left\{\xi_{1}=\xi_{2}<\xi_{3}=\xi_{4}=\xi_{5}\right\} \\
&+\mathbf{P}\left\{\xi_{1}=\xi_{2}>\xi_{3}=\xi_{4}=\xi_{5}\right\}+\mathbf{P}\left\{\xi_{1}=\xi_{2}=\xi_{3}=\xi_{4}<\xi_{5}\right\} \\
&+\mathbf{P}\left\{\xi_{1}=\xi_{2}=\xi_{3}=\xi_{4}>\xi_{5}\right\}+P_{3,2} \\
&=2\left(\mathbf{P}\left\{\xi_{1}=\xi_{2}<\xi_{3}<\xi_{4}=\xi_{5}\right\}+\mathbf{P}\left\{\xi_{1}=\xi_{2}<\xi_{3}=\xi_{4}<\xi_{5}\right\}\right. \\
&\left.+\mathbf{P}\left\{\xi_{1}=\xi_{2}>\xi_{3}=\xi_{4}>\xi_{5}\right\}\right)+P_{3,2}-P_{5}+P_{4}-P_{5}+P_{3,2},
\end{aligned}
$$

что изначально было равно $P_{2,2}$. Из доказанного равенства следует формула для преобразования четвертой группы слагаемых:

$$
\begin{aligned}
\mathbf{P}\left\{\xi_{1}=\xi_{2}<\xi_{3}<\xi_{4}=\xi_{5}\right\} & +\mathbf{P}\left\{\xi_{1}=\xi_{2}<\xi_{3}=\xi_{4}<\xi_{5}\right\} \\
& +\mathbf{P}\left\{\xi_{1}=\xi_{2}>\xi_{3}=\xi_{4}>\xi_{5}\right\}=\left(P_{2,2}-P_{4}\right) / 2-P_{3,2}+P_{5}
\end{aligned}
$$

В пятую группу войдет два слагаемых с коэффициентами $5 ! /(3 ! 2 !)$, и они будут преобразованы с помощью формулы (27).

В группу слагаемых с коэффициентами 5!/4! войдет тоже два слагаемых, и они будут преобразованы с помощью формулы (26).

В седьмую группу войдет единственное последнее слагаемое, и по определению

$$
P_{5}=\mathbf{P}\left\{\xi_{1}=\xi_{2}=\xi_{3}=\xi_{4}=\xi_{5}\right\}
$$

В результате указанных преобразований исходное тождество приобретет вид

$$
\begin{aligned}
1=5 ! & \mathbf{P}\left\{\xi_{1}<\xi_{2}<\xi_{3}<\xi_{4}<\xi_{5}\right\} \\
& +\frac{5 !}{2}\left(\mathbf{P}\left\{\xi_{1}<\xi_{2}<\xi_{3}<\xi_{4}\right\}-5 \mathbf{P}\left\{\xi_{1}<\xi_{2}<\xi_{3}<\xi_{4}<\xi_{5}\right\}\right) \\
& +\frac{5 !}{3 !}\left(\frac{P_{3}-P_{3,2}}{2}-P_{4}+P_{5}\right)+\frac{5 !}{2 \cdot 2}\left(\frac{P_{2,2}-P_{4}}{2}-P_{3,2}+P_{5}\right)+P_{5}
\end{aligned}
$$

Формула (28) получается, если из последнего равенства выразить вероятность $\mathbf{P}\left\{\xi_{1}<\xi_{2}<\xi_{3}<\xi_{4}<\xi_{5}\right\}$, применить формулу (24) и привести подобные члены.

Лемма доказана. 
Лемма 5. Если $\xi_{1}, \xi_{2}, \xi_{3}, \xi_{4}, \xi_{5}, \xi_{6}$ - перестановочные случайные величины, то в соответствии с введенными обозначениями

$$
\mathbf{P}\left\{\xi_{1}<\xi_{2}<\xi_{3}, \xi_{4}<\xi_{5}<\xi_{6}\right\}=\frac{1}{36}-\frac{P_{2}}{6}+\frac{P_{3}}{9}+\frac{P_{2,2}}{4}-\frac{P_{3,2}}{3}+\frac{P_{3,3}}{9} .
$$

Доказательство. Подобно тому, как при доказательстве леммы 1 из представления вероятности полного события

$$
\begin{aligned}
1 & =3 ! \mathbf{P}\left\{\xi_{1}<\xi_{2}<\xi_{3}\right\}+3 \mathbf{P}\left\{\xi_{1}=\xi_{2}<\xi_{3}\right\}+3 \mathbf{P}\left\{\xi_{1}=\xi_{2}>\xi_{3}\right\}+\mathbf{P}\left\{\xi_{1}=\xi_{2}=\xi_{3}\right\} \\
& =6 \mathbf{P}\left\{\xi_{1}<\xi_{2}<\xi_{3}\right\}+3 P_{2}-2 P_{3} .
\end{aligned}
$$

было получено равенство (3), так из аналогичных представлений вероятностей

$$
P_{2}=\mathbf{P}\left\{\xi_{4}=\xi_{5}\right\}, \quad P_{3}=\mathbf{P}\left\{\xi_{4}=\xi_{5}=\xi_{6}\right\}, \quad \mathbf{P}\left\{\xi_{1}<\xi_{2}<\xi_{3}\right\}
$$

получаются равенства

$$
\begin{aligned}
\mathbf{P}\left\{\xi_{1}<\xi_{2}<\xi_{3}, \xi_{4}=\xi_{5}\right\} & =\frac{P_{2}}{6}-\frac{P_{2,2}}{2}+\frac{P_{3,2}}{3}, \\
\mathbf{P}\left\{\xi_{1}<\xi_{2}<\xi_{3}, \xi_{4}=\xi_{5}=\xi_{6}\right\} & =\frac{P_{3}}{6}-\frac{P_{3,2}}{2}+\frac{P_{3,3}}{3}, \\
\mathbf{P}\left\{\xi_{1}<\xi_{2}<\xi_{3}, \xi_{4}<\xi_{5}<\xi_{6}\right\} & =\frac{1}{6} \mathbf{P}\left\{\xi_{1}<\xi_{2}<\xi_{3}\right\}-\frac{1}{2} \mathbf{P}\left\{\xi_{1}<\xi_{2}<\xi_{3}, \xi_{4}=\xi_{5}\right\} \\
& +\frac{1}{3} \mathbf{P}\left\{\xi_{1}<\xi_{2}<\xi_{3}, \xi_{4}=\xi_{5}=\xi_{6}\right\} .
\end{aligned}
$$

Из этих равенств следует утверждение леммы.

\section{4. Доказательства теорем}

Доказательство теоремы 1. Выражение для математического ожидания следует из определения величины $F$ и из формулы (1). Вычислим дисперсию:

$$
\begin{aligned}
\mathbf{D} R= & \sum_{i=1}^{n-1} \mathbf{D} \chi\left\{\xi_{i}<\xi_{i+1}\right\}+2 \sum_{i=1}^{n-3} \sum_{j=i+2}^{n-1} \operatorname{cov}\left(\chi\left\{\xi_{i}<\xi_{i+1}\right\}, \chi\left\{\xi_{j}<\xi_{j+1}\right\}\right) \\
& \quad+2 \sum_{i=1}^{n-2} \operatorname{cov}\left(\chi\left\{\xi_{i}<\xi_{i+1}\right\}, \chi\left\{\xi_{i+1}<\xi_{i+2}\right\}\right) \\
= & (n-\mathrm{i}) D+(n-2)(n-3) c_{4}-2(n-2) c_{\leqslant<}
\end{aligned}
$$

Используя равенства (8), (18) и (10), получаем, что

$$
\begin{aligned}
\mathrm{D} R & =(n-1) \frac{1-P_{2}^{2}}{4}+(n-2)(n-3) \frac{P_{2,2}-P_{2}^{2}}{4}-2(n-1)\left(\frac{1-P_{2}^{2}}{12}-\frac{P_{2}^{2}-P_{3}}{3}\right) \\
& =\frac{n+1}{12}\left(1-P_{2,2}\right)+\frac{2}{3}(n-2)\left(P_{3}-P_{2,2}\right)+\frac{1}{4}(n-1)^{2}\left(P_{2,2}-P_{2}^{2}\right) .
\end{aligned}
$$

Теорема 1 доказана. 
Доказательство теоремы 2 . В силу замечания 3 распределения случайных величин $R$ и $F$ совпадают. Остается доказать формулу для их ковариации. По определению

$$
\begin{aligned}
\operatorname{cov}(R, F)= & \sum_{i=1}^{n-1} \sum_{j=1}^{n-1} \operatorname{cov}\left(\chi\left\{\xi_{i}>\xi_{i+1}\right\}, \chi\left\{\xi_{j}<\xi_{j+1}\right\}\right) \\
= & \sum_{i=1}^{n-1} \operatorname{cov}\left(\chi\left\{\xi_{i}>\xi_{i+1}\right\}, \chi\left\{\xi_{i}<\xi_{i+1}\right\}\right) \\
& +2 \sum_{i=1}^{n-3} \sum_{j=i+2}^{n-1} \operatorname{cov}\left(\chi\left\{\xi_{i}>\xi_{i+1}\right\}, \chi\left\{\xi_{j}<\xi_{j+1}\right\}\right) \\
& +\sum_{i=1}^{n-2} \operatorname{cov}\left(\chi\left\{\xi_{i}>\xi_{i+1}\right\}, \chi\left\{\xi_{i+1}<\xi_{i+2}\right\}\right) \\
& +\sum_{i=1}^{n-2} \operatorname{cov}\left(\chi\left\{\xi_{i}<\xi_{i+1}\right\}, \chi\left\{\xi_{i+1}>\xi_{i+2}\right\}\right) \\
= & -(n-1) D+(n-2)(n-3) c_{4}+(n-2) c_{\leqslant \leqslant}+(n-2) c_{<<.}
\end{aligned}
$$

Для завершения доказательства остается подставить в последнее выражение формулы (8), (18), (11) и (9).

Теорема 2 доказана.

Доказательство теоремы 3. Выражения для математического ожидания и дисперсии случайной величины $L$ получается из теорем 1 и 2 и тождества

$$
R+L+F=n-1 \text {, }
$$

но проще их найти непосредственно. Выражение для математического ожидания следует из определения значения $P_{2}$. Равенство

$$
\mathbf{D} L=-2 \operatorname{cov}(L, R)=-2 \operatorname{cov}(L, F)
$$

получается, если рассмотреть дисперсии правой и левой части тождества

$$
R+L=n-1-F
$$

и учесть равенство распределений $F$ и $R$. Для дисперсии находим выражение

$$
\begin{aligned}
\mathbf{D} L=(n-1) \mathbf{D} \chi\left\{\xi_{1}=\xi_{2}\right\}+2(n-2) \operatorname{cov}\left(\chi\left\{\xi_{1}=\xi_{2}\right\}, \chi\left\{\xi_{2}=\xi_{3}\right\}\right) \\
+(n-2)(n-3) \operatorname{cov}\left(\chi\left\{\xi_{1}=\xi_{2}\right\}, \chi\left\{\xi_{3}=\xi_{4}\right\}\right) .
\end{aligned}
$$

Для завершения доказательства остается применить формулы из следствий 1 и 2 .

Доказательство теоремы 4. По определению

$$
M L^{3}=M \sum_{i, j, k=1}^{n-2} \chi\left\{\xi_{i}=\xi_{i+1}\right\} \chi\left\{\xi_{j}=\xi_{j+1}\right\} \chi\left\{\xi_{k}=\xi_{k+1}\right\}
$$

Отсюда с учетом перестановочности $\xi_{1}, \xi_{2}, \ldots, \xi_{n}$ следует утверждение теоремы 4 . 
Доказательство теоремы 5. По определению

$$
\begin{aligned}
\mathbf{M} \widehat{R R} & =\mathbf{M} \sum_{k=1}^{n-2} \chi\left\{\xi_{k}<\xi_{k+1}<\xi_{k+2}\right\} \\
& =(n-2) \mathbf{P}\left\{\xi_{1}<\xi_{2}<\xi_{3}\right\} .
\end{aligned}
$$

Учитывая (3), получаем первое утверждение теоремы. Чтобы доказать второе утверждение, вычислим $M \widehat{R R}^{2}$. Учитывая перестановочность $\xi_{1}, \xi_{2}, \ldots, \xi_{n}$, находим, что

$$
\begin{aligned}
& \mathbf{M} \widehat{R R}^{2}= \mathbf{M} \sum_{j=1}^{n-2} \chi\left\{\xi_{j}<\xi_{j+1}<\xi_{j+2}\right\} \sum_{k=1}^{n-2} \chi\left\{\xi_{k}<\xi_{k+1}<\xi_{k+2}\right\} \\
&=(n-2) \mathbf{P}\left\{\xi_{1}<\xi_{2}<\xi_{3}\right\}+2(n-3) \mathbf{P}\left\{\xi_{1}<\xi_{2}<\xi_{3}<\xi_{4}\right\} \\
&+(n-4)(n-5) \mathbf{P}\left\{\xi_{1}<\xi_{2}<\xi_{3}, \xi_{4}<\xi_{5}<\xi_{6}\right\} \\
&+2(n-4) \mathbf{P}\left\{\xi_{1}<\xi_{2}<\xi_{3}<\xi_{4}<\xi_{5}\right\} .
\end{aligned}
$$

Отсюда получаем, что

$$
\begin{aligned}
\mathbf{D} \widehat{R R}= & \mathbf{M} \widehat{R R}^{2}-(\mathbf{M} \widehat{R R})^{2}=(n-2) \mathbf{P}\left\{\xi_{1}<\xi_{2}<\xi_{3}\right\} \\
& +2(n-3) \mathbf{P}\left\{\xi_{1}<\xi_{2}<\xi_{3}<\xi_{4}\right\}+2(n-4) \mathbf{P}\left\{\xi_{1}<\xi_{2}<\xi_{3}<\xi_{4}<\xi_{5}\right\} \\
& +(n-4)(n-5) \mathbf{P}\left\{\xi_{1}<\xi_{2}<\xi_{3}, \xi_{4}<\xi_{5}<\xi_{6}\right\}-(n-2)^{2} \mathbf{P}^{2}\left\{\xi_{1}<\xi_{2}<\xi_{3}\right\} .
\end{aligned}
$$

Используя формулы лемм 1-5, путем элементарных преобразований выводим второе утверждение теоремы 5.

Доказательство теоремы 6. По определению

$$
\begin{aligned}
\mathbf{M} \widehat{L L} & =\mathbf{M} \sum_{k=1}^{n-2} \chi\left\{\xi_{k}=\xi_{k+1}=\xi_{k+2}\right\} \\
& =(n-2) \mathbf{P}\left\{\xi_{1}=\xi_{2}=\xi_{3}\right\} .
\end{aligned}
$$

Учитывая определение $P_{3}$, получаем первое утверждение теоремы. Чтобы доказать второе утверждение, вычислим $\mathbf{M} \widehat{L L}^{2}$. Учитывая перестановочность $\xi_{1}, \xi_{2}, \ldots, \xi_{n}$, находим, что

$$
\begin{aligned}
& \mathbf{M} \widehat{L L}^{2}= \mathbf{M} \sum_{j=1}^{n-2} \chi\left\{\xi_{j}=\xi_{j+1}=\xi_{j+2}\right\} \sum_{k=1}^{n-2} \chi\left\{\xi_{k}=\xi_{k+1}=\xi_{k+2}\right\} \\
&=(n-2) \mathbf{P}\left\{\xi_{1}=\xi_{2}=\xi_{3}\right\}+2(n-3) \mathbf{P}\left\{\xi_{1}=\xi_{2}=\xi_{3}=\xi_{4}\right\} \\
& \quad+2(n-4) \mathbf{P}\left\{\xi_{1}=\xi_{2}=\xi_{3}=\xi_{4}=\xi_{5}\right\} \\
& \quad+(n-4)(n-5) \mathbf{P}\left\{\xi_{1}=\xi_{2}=\xi_{3}, \xi_{4}=\xi_{5}=\xi_{6}\right\} .
\end{aligned}
$$

Отсюда получаем равенство

$$
\begin{aligned}
\mathbf{D} \widehat{L L} & =\mathbf{M} \widehat{L L}^{2}-(\mathbf{M} \widehat{L L})^{2} \\
& =(n-2) P_{3}+2(n-3) P_{4}+2(n-4) P_{5}+(n-4)(n-5) P_{3,3}-(n-2)^{2} P_{3}^{2},
\end{aligned}
$$

которое после элементарных преобразований дает нужную формулу для дисперсии.

Теорема 6 доказана. 
Доказательство теоремы 7. Выражение для математического ожидания числа пиков следует из формулы (4). Выражение для математического ожидания числа впадин следует из формулы (5).

Теорема 7 доказана.

\section{Список литературы}

1. Зубков А. М., Шуваев Д. В., Первые моменты числа отрезков неубывания в последовательности перестановочных случайных величин. Обозрение прикладной и промышленной математики (2001) 8, №2, 760-761.

2. Риордан Дж., Введение в комбинаторный анализ. ИЛ, Москва, 1963.

3. Сачков В. Н., Введение в комбинаторные методы дискретной математики. Наука, Москва, 1983.

4. Yi-Liang Chen, Wei-Hou Cheng, A note on joint distributions of some random vectors defined on permutations. Ars Combinatoria (2000) 54, 139-148.

Статья поступила 02.12.2004. 